\title{
Contracaecum multipapillatum (Nematoda: Anisakidae) en pecesdeaguascontinentales de Guanacaste, Costa Rica e Izabal, Guatemala
}

\author{
Contracaecum multipapillatum (Nematoda: Anisakidae) in inland \\ water fish from Guanacaste, Costa Rica and Izabal, Guatemala
}

Choc-ML, Jiménez-RA, Arguedas-CD, Dolz G. Contracaecum multipapillatum (Nematoda: Anisakidae) en peces de aguas continentales de Guanacaste, Costa Rica e Izabal, Guatemala. Rev Colombiana Cienc Anim. Recia. 2020; 12(2):e767. https://doi.org/10.24188/recia.v12.n2.2020.767

Universidad de Sucre, Colombia

Los autores permiten a RECIA reimprimir el material publicado en él. En caso de que un autor quiera traducir o usar una publicación parcial o completa de nuestro Diario, el autor debe obtener un permiso por escrito del editor de la revista.

Copyright (C) 2020. El (los) autor (es), Revista Colombiana de Ciencia Animal - RECIA. 2020. Este es un artículo de acceso abierto distribuido bajo los términos de Creative Commons Attribution 4.0 (https://creativecommons.org/licenses/by-nc-sa/4.0/), El uso, distribución o reproducción está permitido, siempre que se acrediten al autor original y al propietario del copyright y que se cite la publicación original en esta revista, de acuerdo con la práctica académica aceptada. No se permite el uso, distribución o reproducción que no cumpla con estos términos. 


\title{
Contracaecummultipapillatum(Nematoda:Anisakidae) en peces de aguas continentales de Guanacaste, Costa Rica e Izabal, Guatemala
}

\author{
Contracaecum multipapillatum (Nematoda: Anisakidae) in inland water fish from \\ Guanacaste, Costa Rica and Izabal, Guatemala
}

Luis Choc-M. M.Sc.

Universidad Nacional, Posgrado en Ciencias Veterinarias Tropicales, Maestría en Enfermedades Tropicales, Heredia, Costa Rica.

Universidad de San Carlos de Guatemala, Facultad de Medicina

Veterinaria y Zootecnia, Unidad de Parasitología, Guatemala.

*luischoc25@gmail.com

(D) https://orcid.org/0000-0002-0917-8011

Ana Jiménez-R. Ph.D.

Universidad Nacional, Posgrado en Ciencias Veterinarias Tropicales,

Maestría en Enfermedades Tropicales, Heredia, Costa Rica.

ana.jimenez.rocha@una.cr

(iD) https://orcid.org/0000-0001-5416-9029

Donald Arguedas-C. Ph.D.

Universidad Técnica Nacional, Laboratorio para Aguas y Reproducción de Especies Dulceacuícolas, Guanacaste, Costa Rica.

darguedas@utn.ac.cr

(D) https://orcid.org/0000-0002-0127-4754

Gaby Dolz. Ph.D.

Universidad Nacional, Posgrado en Ciencias Veterinarias Tropicales,

Maestría en Enfermedades Tropicales, Heredia, Costa Rica.

Recepción: 12 mayo 2020

gaby.dolz.wiedner@una.ac.cr

(1) https://orcid.org/0000-0002-9566-5130

\section{RESUMEN}

Contracaecum multipapillatum es un nemátodo para el cual, los peces actúan como hospedador intermediario y el ser humano como hospedador accidental, por consumo de carne de pescado cruda o semi cocinada. Este riesgo para la salud humana hace importante determinar su prevalencia, intensidad de infección y ubicación anatómica en peces destinados al consumo mediante pesca artesanal; y la influencia de parámetros físicoquímicos del agua sobre la infección en peces. Se recolectaron 214 peces en 6 ríos de Cañas, Guanacaste, Costa Rica y 203 peces en el Lago de Izabal y Río Dulce, Izabal, Guatemala. En los sitios de pesca se tomaron parámetros físicoquímicos del agua (temperatura, pH y oxígeno disuelto). La prevalencia de infección fue 16.8\% y 25.1\% en Guanacaste e Izabal, respectivamente, encontrándose 105 larvas de tercer estadio (L3) en 36 peces de Guanacaste y 135 L3 en 51 peces de Izabal. Se observó afinidad de L3 por órganos viscerales (22.4\% en Guanacaste - 25.1\% en Izabal) y menor por tejido muscular (2.0\% en Guanacaste - 1.0\% en Izabal). La intensidad de infección fue directamente proporcional con la talla y peso de los peces (una larva en 13 peces con peso y talla media de $66.7 \mathrm{~g}$ y $17.5 \mathrm{~cm}$ en Guanacaste, y siete peces de $214.9 \mathrm{~g}$ y $28.4 \mathrm{~cm}$ en Izabal; y 14 larvas en un pez de 148.8 g y $24 \mathrm{~cm}$ en Guanacaste y 12 larvas en un pez de 260 y y $40 \mathrm{~cm}$ en Izabal). No se observó asociación entre parámetros fisicoquímicos del agua y susceptibilidad de peces a infección.

Palabras clave: Parásitos; Contracecosis; zoonosis; infección; pesca artesanal; prevalencia. 


\begin{abstract}
Contracaecum multipapillatum is a nematode where the fish act as an intermediate host and the human being as an accidental host, by consuming raw or semi-cooked fish meat, causing Contracecosis. This risk to human health makes important to determine prevalence, infection intensity and anatomical location in fish for human consumption through artisanal fishing; and the physicochemical parameters influence of water on infection in fish. 214 fish were collected in 6 rivers from Cañas, Guanacaste, Costa Rica and 203 fish in Izabal Lake and Río Dulce, Izabal, Guatemala. Water physicochemical parameters (temperature, $\mathrm{pH}$ and dissolved oxygen) were taken at fishing sites. The infection prevalence was $16.8 \%$ and $25.1 \%$ in Guanacaste and Izabal, respectively, 36 fish were found infected with 105 larvae of the third stage (L3) in Guanacaste and 135 larvae were found in 51 infected fish in Izabal. A higher affinity of L3 was observed in visceral organs $(22.4 \%$ in Guanacaste $-25.1 \%$ in Izabal) and lower affinity in muscle tissue $(2.0 \%$ in Guanacaste - $1.0 \%$ in Izabal). Infection intensity was proportional to the size and weight fish (1 larva in 13 fish with average weight and size of $66.7 \mathrm{~g}$ and $17.5 \mathrm{~cm}$ in Guanacaste, and in $7 \mathrm{fish}$ with weight and average size of $214.9 \mathrm{~g}$ and $28.4 \mathrm{~cm}$ in Izabal; while 14 larvae were found in a fish of $148.8 \mathrm{~g}$ and $24 \mathrm{~cm}$ in Guanacaste and 12 larvae in a fish of $260 \mathrm{~g}$ and $40 \mathrm{~cm}$ in Izabal). No association was observed between the water physicochemical parameters and the fish susceptibility to infection.
\end{abstract}

Keywords: Parasites; Contracecosis; zoonoses; infection; artisanal fishing; prevalence.

\title{
INTRODUCCIÓN
}

Los nematodos de la familia Anisakidae comprenden al menos nueve géneros, de los cuales Anisakis, Pseudoterranova y Contracaecum, han cobrado importancia por producir infecciones zoonóticas al consumir carne de pescado cruda o insuficientemente cocinada (1). La especie Contracaecum multipapillatum (Von Drasche 1882) posee un ciclo de vida que se desarrolla en ambientes acuáticos, parasitando a diferentes especies de crustáceos en sus primeras fases larvarias y a peces en su tercer estadio larvario, siendo esta la fase infectante para los hospederos definitivos que son aves piscívoras, en las que se da la reproducción sexual y liberación de huevos hacia el medio ambiente acuático $(2,3,4)$.

El género Contracaecum ha sido reportado en cíclidos del género Parachromis sp. (Guapotes) en Perú (5), mientras que Mancinni et al (2) reportaron prevalencias de Contracaecum spp. del 70 y 87\% en Hoplias spp. (Guabinas) y Rhamdia spp. (Barbudos o bagres) de Argentina, y Maniscalchi et al (6) establecieron prevalencias de 83.6\% en peces del género Mugil spp. (Lisas) de Venezuela, siendo estos los únicos reportes en América Latina de hallazgos de C. multipapillatum en peces destinados al consumo humano.

Los reportes que asocian los nemátodos anisákidos con infecciones accidentales en el ser humano son también escasos en Latinoamérica (7). En Perú se confirmó la infección por nemátodos de los géneros Anisakis y Pseudoterranova (7), en Argentina se reportaron infecciones por Pseudoterranova sp. en seres humanos (8), mientras que en algunos casos no se determinó el género y especie de anisákido involucrado (9). Aunque la capacidad infectiva de Contracaecum spp. no ha sido confirmada en seres humanos debido a la ausencia de reportes de infecciones específicas, si ha sido comprobada experimentalmente en modelos animales en México y Brasil (5).

La infección en el ser humano es conocida como Contracecosis y clínicamente produce tres etapas características que se desarrollan según la capacidad de la larva de superar la respuesta inmune del ser humano (1). La primera etapa se caracteriza por signos gastro alérgicos que inician con vómitos y diarrea acompañados de dolor agudo por penetración de la larva infectiva en la mucosa gástrica o duodenal, la segunda etapa se da por la migración extraintestinal de la larva originando un cuadro clínico variable según el órgano afectado, y la tercera es de tipo crónico o gastro alérgico, caracterizado por reacciones alérgicas en piel o en tracto respiratorio que se manifiestan como procesos asmáticos $(3,10)$.

Debido al hallazgo y confirmación de la presencia de Contracaecum multipapillatum s.l. en peces comerciales de Guanacaste, Costa Rica e Izabal, Guatemala, mediante análisis morfológico y molecular, cobra importancia conocer y determinar los factores que influyen en la presencia de estos nemátodos en determinadas poblaciones de peces y el riesgo que pueden representar para la salud humana, por lo que este estudio planteó como objetivo determinar la prevalencia de infección del parasito y la intensidad de infección en peces de consumo humano (Guapotes, Barbudos y Guabinas) en distintos sitios de pesca artesanal de Guanacaste y Guatemala, así como la ubicación de la larva 3 (L3) en los peces y correlacionar su presencia con parámetros fisicoquímicos del medio ambiente acuático. 


\section{METERIALES Y MÉTODOS}

Áreas de estudio. Entre enero y julio de 2017 se recolectaron peces en seis ríos de la provincia de Guanacaste Costa Rica, y cinco puntos del Lago de Izabal y Río Dulce, del departamento de Izabal, Guatemala todos ellos cercanos a comunidades que practican pesca artesanal.

Los ríos en los cuales se desarrolló el muestreo en Guanacaste fueron el Canal Interno de la Finca de la Universidad Técnica Nacional (UTN), los ríos Duque, Paso Hondo, Cañas, Higuerón y Abangares, todos ubicados en el cantón de Cañas (Figura 1). Los sitios de muestreo seleccionados en Izabal fueron puntos de pesca cercanos a las comunidades de El Estor y Mariscos, ambos dentro del Lago de Izabal; y las comunidades de Río Dulce, El Higuerito y Cayo Piedra, situadas dentro de la cuenca de Río Dulce. En ambas regiones se tomaron coordenadas de los sitios de muestreo con equipo de georreferenciación (Figura 2).

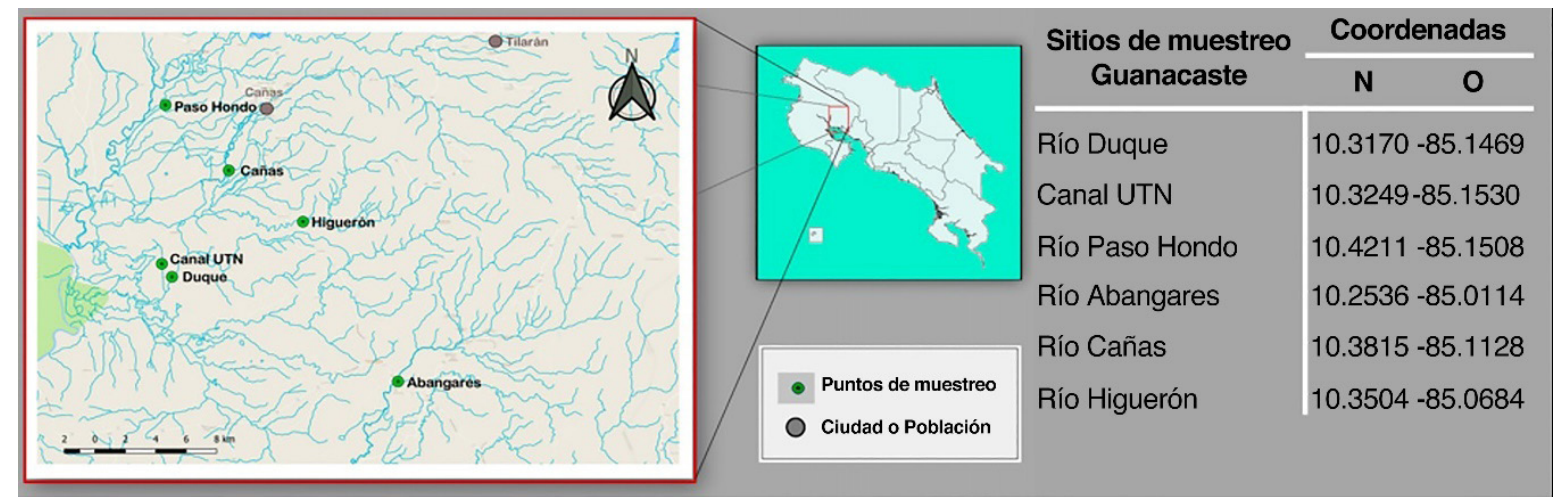

Figura 1. Mapa de coordenadas geográficas donde se llevaron a cabo los muestreos en la provincia de Guanacaste, Costa Rica.

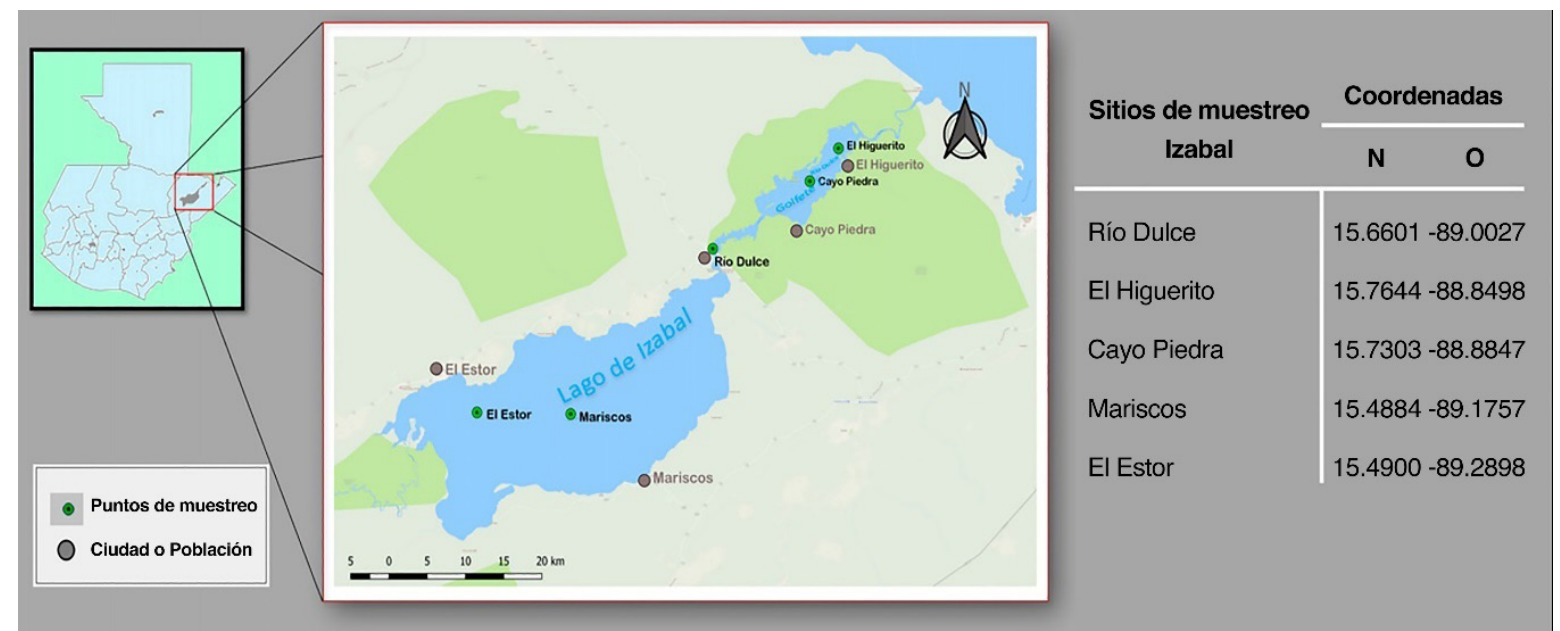

Figura 2. Mapa de coordenadas geográficas donde se llevaron a cabo los muestreos en el departamento de Izabal, Guatemala.

Tamaño de muestra: El tamaño de muestra para determinar la prevalencia de la infección (nivel de confianza 95\%, error aceptado 7.5\%, prevalencia esperada 31\%) se calculó mediante el programa WinEpiscope, utilizando parámetros para población infinita. Se recolectaron 214 y 203 peces en Guanacaste e Izabal, respectivamente.

Recolecta de datos y peces: Los peces capturados se transportaron en frio para su posterior análisis.

Al momento de la pesca se tomaron parámetros fisicoquímicos del medio ambiente acuático ( $\mathrm{pH}$, temperatura y oxígeno) por medio de una sonda multiparamétrica en cada sitio de pesca.

En el laboratorio se tomaron datos biométricos de peso y tamaño de cada pez y se identificaron taxonómicamente a nivel de género, seguidamente fueron sacrificados mediante congelamiento, y se realizó la necropsia buscando larvas de 
diferentes especies de parásitos anisákidos en órganos viscerales y musculatura axial. Para establecer la intensidad de infección por región anatómica se colocaron los nemátodos extraídos en cajas de Petri, y se realizó el conteo del número de larvas extraídas por pez y por ubicación (vísceras o músculo).

Análisis parasitológico: Las larvas extraídas (105 de Guanacaste y 135 de Izabal) se sometieron a digestión artificial en pepsina 1.5\%, ácido clorhídrico 0.8\% (concentración al 25\%), en agitación constante a $42^{\circ} \mathrm{C}$ hasta lograr la liberación de las larvas de los tejidos, y se preservaron en alcohol al 70\% hasta su diagnóstico mediante microscopía óptica y técnicas moleculares como se describió previamente por Solas et al. (11).

Los géneros de larvas se determinaron mediante microscopía óptica, basándose en características anatómicas internas del género Contracaecum, como lo son la presencia y ubicación del ciego paraesofágico, esófago, ventrículo y apéndice ventricular; y características externas como la presencia de diente apical, labios y mucrón de acuerdo con las características morfológicas reportadas por Silva et al (12). Todas las larvas se determinaron pertenecientes al género Contracaecum.

Análisis molecular: La especie de las larvas se confirmó amplificando un segmento de 519 pb del gen mitocondrial de la Citocromo Oxidasa 2 (COX2) de acuerdo con las indicaciones del protocolo descrito por D’Amelio et al. (13), utilizando los iniciadores 211F (5'-TTT TCT AGT TAT ATA GAT TGR TTY AT-3') y 210R (5'-CAC CAA CTC TTA AAA TTA TC-3'). El protocolo de amplificación fue: desnaturalización inicial a $94^{\circ} \mathrm{C}$ durante 3 minutos, 34 ciclos de desnaturalización $\left(94^{\circ} \mathrm{C}\right.$ durante 30 segundos), alineación $\left(46^{\circ} \mathrm{C}\right.$ durante 30 segundos) y extensión $\left(72^{\circ} \mathrm{C}\right.$ durante 90 segundos) y una extensión final a $72^{\circ} \mathrm{C}$ durante 7 minutos. Los productos amplificados se enviaron a secuenciar a Macrogen, Korea.

Análisis estadístico: Se identificaron las zonas de muestreo con presencia de peces infectados, se estableció la prevalencia de infección (peces infectados/total peces analizados x 100) y la intensidad de infección (número de individuos de una especie particular de parásito en un hospedero infectado) (14). Se determinó además los géneros de peces infectados, la ubicación anatómica (musculo, vísceras) de las larvas y los parámetros fisicoquímicos (pH, temperatura y oxígeno) de los sitios de pesca, para determinar diferencias significativas con respecto a la intensidad de infección utilizando el programa Win epi ${ }^{\circledR}$. Para ello se calcularon los valores de $\mathrm{Chi}^{2}$ para prueba de dos colas o prueba corregida de Yates en muestras de tamaño no proporcional.

\section{RESULTADOS}

Se capturaron tres tipos de peces mediante pesca artesanal: Parachromis sp., (Guapote) Rhamdia sp. (Barbudo) y Hoplias sp. (Guabina). Las larvas de tercer estadio (L3) extraídas se identificaron como pertenecientes al género Contracaecum mediante microscopía óptica. Las larvas mostraron una longitud media de $17 \mathrm{~mm}$, las estructuras observadas en el extremo cefálico correspondieron a un diente apical de 0.02 a $0.025 \mathrm{~mm}$ de longitud, mientras que a lo largo del tercio anterior de la larva se observó un esófago recto (3-3.2 mm) acompañado por un ciego paraesofágico de 2 a $2.5 \mathrm{~mm}$ de longitud, tanto esófago como ciego paraesofágico desembocaron en un ventrículo al final del tercio anterior que midió $0.1 \mathrm{~mm}$, desde el cual se extendió en dirección ventral y caudal un apéndice ventricular de $0.4 \mathrm{~mm}$ de longitud y en dirección media caudal el tubo intestinal. En el extremo ventral caudal se observó el orificio anal y en el extremo final caudal una estructura terminal aguda conocida como mucrón que midió de 0.04 a 0.05 mm de longitud. La amplificación y secuenciación del gen COX II determinó a las L3 de Costa Rica y Guatemala pertenecientes a la especie C. multipapillatum s.l., mostrando una identidad nucleotídica del 88-94\% con muestras de C. multipapillatum aisladas en los Estados Unidos (AF179910) y Grecia (EU852344). Las secuencias de C. multipapillatum de Costa Rica y Guatemala se depositaron en GenBank bajo los números de acceso MH044672-MH044685.

Se estableció una prevalencia de infección de 16.8\% (36/214) en peces de Guanacaste y 25.1\% (51/203) en peces de Izabal. Los sitios con presencia de peces infectados en Guanacaste fueron los ríos Abangares, Cañas e Higuerón, mientras que, en Izabal, se encontraron peces infectados con larvas de C. multipapillatum s.l. en todos los sitios muestreados. El porcentaje de infección en peces de los diferentes sitios de muestreo tanto de Guanacaste como de Izabal se muestra en la Tabla 1. 
Tabla 1. Número de diferentes especies de peces (Rhamdia sp., Parachromis sp. y Hoplias sp.) capturadas en los diferentes sitios de muestreo en Guanacaste, Costa Rica e Izabal, Guatemala y porcentaje de infección con C. multipapillatum.

\begin{tabular}{|c|c|c|c|c|}
\hline \multirow{2}{*}{ Sitio de muestreo Guanacaste } & Rhamdia sp. & Parachromis sp. & Hoplias sp. & Total \\
\hline & $\%$ (+/total) & $\%$ (+/total) & $\%$ (+/total) & $\%$ (+/total) \\
\hline Río Duque & $0(0 / 1)$ & $0(0 / 1)$ & $0(0 / 0)$ & $0(0 / 2)$ \\
\hline Canal UTN & $0(0 / 0)$ & $0(0 / 2)$ & $0(0 / 0)$ & $0(0 / 2)$ \\
\hline Río Paso Hondo & $0(0 / 20)$ & $0(0 / 26)$ & $0(0 / 0)$ & $0(0 / 46)$ \\
\hline Río Abangares & $0(0 / 1)$ & $29.4(5 / 17)$ & $100(1 / 1)$ & $31.6(6 / 19)$ \\
\hline Río Cañas & $13.5(5 / 37)$ & $17.0(8 / 47)$ & $0(0 / 0)$ & $15.5(13 / 84)$ \\
\hline Río Higuerón & $45.4(5 / 11)$ & $24.0(12 / 50)$ & $0(0 / 0)$ & $27.9(17 / 61)$ \\
\hline TOTAL & $14.3(10 / 70)$ & $17.5(25 / 143)$ & $100(1 / 1)$ & $16.8(36 / 214)$ \\
\hline \multirow{2}{*}{ Sitio de muestreo Izabal } & Rhamdia sp. & Parachromis sp. & Hoplias sp. & Total \\
\hline & $\%$ (+/total) & $\%$ (+/total) & $\%$ (+/total) & $\%(+/$ total $)$ \\
\hline Río Dulce & $33.3(7 / 21)$ & $41.5(17 / 41)$ & $0(0 / 0)$ & $38.7(24 / 62)$ \\
\hline El Higuerito & $18.2(6 / 33)$ & $20(2 / 10)$ & $0(0 / 0)$ & $18.6(8 / 43)$ \\
\hline Cayo Piedra & $6.4(2 / 31)$ & $0(0 / 0)$ & $0(0 / 0)$ & $6.4(2 / 31)$ \\
\hline Mariscos & $36.4(12 / 33)$ & $0(0 / 0)$ & $0(0 / 0)$ & $36.4(12 / 33)$ \\
\hline El Estor & $14.3(1 / 7)$ & $14.8(4 / 27)$ & $0(0 / 0)$ & $14.7(5 / 34)$ \\
\hline TOTAL & $22.4(28 / 125)$ & $29.5(23 / 78)$ & $0(0 / 0)$ & $25.1(51 / 203)$ \\
\hline
\end{tabular}

La prueba corregida de Yates no determinó diferencias significativas entre los tres sitios de muestreo de Guanacaste con respecto a la presencia de peces parasitados ( $p>0.05)$, mientras que para Izabal se determinó una diferencia significativa entre Cayo Piedra y los demás sitios de muestreo, ya que ahí se encontraron menos peces parasitados con relación a la cantidad de peces recolectados $(\mathrm{p}<0.05)$.

El peso medio de los peces de Guanacaste fue de $92.3 \mathrm{~g}$ y la longitud media fue de $19.0 \mathrm{~cm}$, mientras que el peso medio de los peces de Izabal fue de 351.9 g y la longitud media de $33.9 \mathrm{~cm}$ (Tabla 2). La mayor intensidad de infección determinada en Guanacaste fue de 14 larvas en un espécimen del género Parachromis con peso de 148.8 g y longitud de 24 cm; mientras que en Izabal la mayor intensidad de infección se observó en un pez del género Rhamdia con peso de 260 g y $40 \mathrm{~cm}$ de longitud, que poseía un total de 12 larvas (Tabla 2), siendo estos los peces de mayor talla y peso del total de especímenes.

En Guanacaste la mayor proporción de peces parasitados fue del género Parachromis sp. en el río Abangares (29.4\%), y del género Rhamdia sp. en el río Higuerón (45.4\%) (Tabla 2). Por su parte en Izabal la mayoría de los peces parasitados fue del género Parachromis sp. en Rio Dulce (41.5\%) y del género Rhamdia sp. (36.4\%) en el sitio de Mariscos (Tabla 1). No se determinó diferencias significativas entre infección por C. multipapillatum y género de pez (p>0.05) (Tabla 2).

Se observaron larvas enquistadas en vísceras de los 36 peces parasitados recolectados en Guanacaste, incluyendo el único pez del género Hoplias procedente del río Abangares, mientras que solamente un espécimen perteneciente al género Parachromis proveniente del río Higuerón (3\%), mostró larvas enquistadas en la fascia muscular celómica (Tabla 3). En Izabal también se observaron larvas enquistadas en vísceras de todos los peces parasitados, mientras que dos de estos peces (un Rhamdia y un Parachromis) también mostraron larvas enquistadas en la fascia muscular celómica (Tabla 3). Se estableció una marcada preferencia de las L3 de C. multipapillatum s.l. por vísceras y no por musculatura, tanto en los peces de Guanacaste como de Izabal $(\mathrm{p}<0.05)$ (Tabla 3).

Los parámetros fisicoquímicos del agua (temperatura, $\mathrm{pH}$, oxígeno) en los sitios con presencia de peces parasitados no mostraron diferencias significativas, tanto con la prueba corregida de Yates como con el cálculo de $\mathrm{Chi}^{2}(\mathrm{p}>0.05)$, mientras que los sitios en los que no se encontraron peces parasitados destacaron por sus bajos niveles de oxígeno disuelto en agua $(<4 \mathrm{ppm})$ a diferencia de los sitios con peces parasitados, que presentaron niveles normales de oxígeno (Tabla 4). 
Tabla 2. Intensidad de infección de la L3 de C. mulipapillatum en diferentes especies de peces recolectadas en Guanacaste, Costa Rica (A) e Izabal, Guatemala (B).

\begin{tabular}{|c|c|c|c|c|c|}
\hline $\begin{array}{c}\text { Número de L3 de } C \text {. } \\
\text { multipapillatum }\end{array}$ & $\begin{array}{l}\text { Rhamdia sp. } \\
\% \text { (+/total) }\end{array}$ & $\begin{array}{c}\text { Parachromis sp. } \\
\% \text { (+/total) }\end{array}$ & $\begin{array}{c}\text { Total } \\
\% \text { (+/total) }\end{array}$ & $\begin{array}{c}\text { Peso } \\
\text { Media (g) } \\
\end{array}$ & $\begin{array}{c}\text { Longitud } \\
\text { Media }(\mathrm{cm})\end{array}$ \\
\hline \multicolumn{6}{|c|}{ Costa Rica } \\
\hline 1 & $13.9(5 / 36)$ & $22.2(8 / 36)$ & $36.1(13 / 36)$ & 66.7 & 17.5 \\
\hline 2 & $2.8(1 / 36)$ & $13.9(5 / 36)$ & $16.7(6 / 36)$ & 119.8 & 19.0 \\
\hline 3 & $5.6(2 / 36)$ & $13.9(5 / 36)$ & $19.4(7 / 36)$ & 77.8 & 17.5 \\
\hline 4 & $2.8(1 / 36)$ & $8.3(3 / 36)$ & $13.9(5 / 36)$ & 82.8 & 19.1 \\
\hline 5 & $0(0 / 36)$ & $2.8(1 / 36)$ & $2.8(1 / 36)$ & 77.4 & 18.0 \\
\hline 6 & $2.8(1 / 36)$ & $2.8(1 / 36)$ & $5.6(2 / 36)$ & 72.3 & 18.2 \\
\hline 9 & $0(0 / 36)$ & $2.8(1 / 36)$ & $2.8(1 / 36)$ & 93.0 & 19.0 \\
\hline 14 & $0(0 / 36)$ & $2.8(1 / 36)$ & $2.8(1 / 36)$ & 148.8 & 24.0 \\
\hline Total & $27.8(10 / 36)$ & $69.4(25 / 36)$ & $100(36 / 36)$ & & \\
\hline Media & & & & 92.32 & 19.04 \\
\hline \multicolumn{6}{|c|}{ Guatemala } \\
\hline 1 & $9.8(5 / 51)$ & $3.9(2 / 51)$ & $13.7(7 / 51)$ & 214.9 & 28.4 \\
\hline 2 & $39.2(20 / 51)$ & $17.6(9 / 51)$ & $58.7(29 / 51)$ & 212.9 & 27.8 \\
\hline 3 & $11.8(6 / 51)$ & $3.9(2 / 51)$ & $15.7(8 / 51)$ & 233.2 & 29.2 \\
\hline 4 & $2(1 / 51)$ & $0(0 / 0)$ & $2(1 / 51)$ & 265.0 & 30.0 \\
\hline 5 & $5.9(3 / 51)$ & $0(0 / 0)$ & $5.9(3 / 51)$ & 304.0 & 32.7 \\
\hline 7 & $2(1 / 51)$ & $0(0 / 0)$ & $2(1 / 51)$ & 1045.0 & 48.0 \\
\hline 8 & $2(1 / 51)$ & $0(0 / 0)$ & $2(1 / 51)$ & 280.0 & 35.0 \\
\hline 12 & $2(1 / 51)$ & $0(0 / 0)$ & $2(1 / 51)$ & 260.0 & 40.0 \\
\hline Total & $74.5(38 / 51)$ & $25.5(13 / 51)$ & $100(51 / 51)$ & & \\
\hline Media & & & & 351.87 & 33.89 \\
\hline
\end{tabular}

Tabla 3. Detección de la L3 de C. multipapillatum en vísceras de diferentes especies de peces de Guanacaste, Costa Rica (A) e Izabal, Guatemala (B).

\begin{tabular}{|c|c|c|}
\hline \multirow{2}{*}{ Sitio de muestreo } & \multicolumn{2}{|c|}{ Vísceras } \\
\hline & Rhamdia sp. +/total (\%) & Parachromis sp.+/total (\%) \\
\hline \multicolumn{3}{|c|}{ Costa Rica } \\
\hline Abangares & $0 / 0(0)$ & $5 / 5(100)$ \\
\hline Cañas & $5 / 5(100)$ & $8 / 8(100)$ \\
\hline Higuerón & $5 / 5(100)$ & $11 / 12(92)$ \\
\hline TOTAL & $10 / 10(100)$ & $24 / 25$ (96) \\
\hline \multicolumn{3}{|c|}{ Guatemala } \\
\hline El Estor & $1 / 1(100)$ & $4 / 4(100)$ \\
\hline Río Dulce & $7 / 7(100)$ & $17 / 17(100)$ \\
\hline Mariscos & $12 / 12(100)$ & $0 / 0(0)$ \\
\hline El Higuerito & $6 / 6(100)$ & $2 / 2(100)$ \\
\hline Cayo Piedra & $2 / 2(100)$ & $0 / 0(0)$ \\
\hline TOTAL & $28 / 28(100)$ & $100(23 / 23)$ \\
\hline
\end{tabular}


Tabla 4. Parámetros fisicoquímicos establecidos en los sitios de muestreo de Guanacaste, Costa Rica (A) e Izabal, Guatemala (B) y cantidad de peces parasitados o no parasitados con L3 de C. multipapillatum.

\begin{tabular}{|c|c|c|c|c|c|}
\hline \multirow{2}{*}{ Sitio de muestreo Guanacaste } & \multirow{2}{*}{ Temperatura $\left({ }^{\circ} \mathrm{C}\right)$} & \multirow{2}{*}{$\mathrm{pH}(6.5-8) * *$} & \multirow{2}{*}{ Oxígeno (5-14 mg/l) ** } & \multicolumn{2}{|c|}{ Número de peces } \\
\hline & & & & parasitados & no parasitados \\
\hline \multicolumn{6}{|c|}{ Costa Rica } \\
\hline Río Duque & 25.9 & 7.4 & 2.9 & 0 & 2 \\
\hline Canal Finca UTN & 27.7 & 7.2 & 2.7 & 0 & 2 \\
\hline Río Paso Hondo & 27.7 & 7.3 & 2.7 & 0 & 46 \\
\hline Río Abangares & 30.1 & 8.3 & 4.9 & 6 & 12 \\
\hline Rio Cañas & 28.3 & 7.5 & 6.6 & 13 & 71 \\
\hline Río Higuerón & 28.1 & 7.4 & 6.1 & 17 & 44 \\
\hline TOTAL & & & & 36 & 178 \\
\hline \multicolumn{6}{|c|}{ Guatemala } \\
\hline Río Dulce & 30.4 & 7.9 & 6.5 & 24 & 38 \\
\hline El Higuerito & 30.9 & 8.5 & 7.0 & 8 & 35 \\
\hline Cayo Piedra & 31.0 & 8.8 & 7.0 & 2 & 29 \\
\hline Mariscos & 30.5 & 8.6 & 6.6 & 12 & 21 \\
\hline El Estor & 30.2 & 10.5 & 6.5 & 5 & 29 \\
\hline \multicolumn{2}{|l|}{ TOTAL } & & & 51 & 152 \\
\hline
\end{tabular}

** Datos basados en Boyd, 2015. (19, 20, 21)

\section{DISCUSIÓN}

Las prevalencias de L3 de C. multipapillatum determinadas en Guanacaste (16.8\%) e Izabal (25.1\%) fueron menores a las reportadas en regiones de similar latitud tropical en Latinoamérica, sin embargo, estos datos concuerdan con las observaciones de los estudios realizados en Brasil, (15) y México (3), con respecto a la diferencia entre prevalencias de los diferentes géneros de peces según las características del hábitat acuático, comportamiento ecológico y hábitos alimenticios. En este estudio no se determinó estadísticamente una afinidad específica o una mayor susceptibilidad de alguna de las especies de peces estudiadas a la infección por C. multipapillatum, pero se observó en las dos regiones una mayor infección en Parachromis sp. (Perciformes: Cichlidae) en comparación a Rhamdia sp. (Siluriformes: Heptapteridae), posiblemente debido a que fue la especie que se capturó en mayor número.

Las diferencias en las prevalencias detectadas en Guanacaste e Izabal podrían estar influenciadas por factores medioambientales climáticos. En Guanacaste predomina el clima cálido con escaza presencia de lluvias durante el año, mientras que en Izabal es una región cálida húmeda, con abundante presencia de lluvias durante todo el año. Estos factores pueden influir en las características fisicoquímicas del medio ambiente acuático y favorecer o limitar la presencia de determinadas especies de aves piscívoras, hospederos definitivos o de otros organismos acuáticos que participan en el ciclo de vida de C. multipapillatum $(3,4)$.

Los altos porcentajes de positividad determinados en algunos puntos de muestreo de Guatemala pueden deberse, además, a la cercanía de puntos de comercialización de peces en esas zonas urbanas, y los hábitos relacionados con limpieza y eliminación de vísceras de peces hacia el río. Algunas legislaciones observadas principalmente en países europeos, principalmente en España con el Decreto Real 1420/2006 y en Italia con la Nota Ministerial DGISAN 0024111-P del Ministerio de Salud Italiano (16) que recomiendan el adecuado manejo de vísceras post captura, para evitar reinfección por eliminación de estas en el medio acuático, perpetuando el ciclo biológico de los anisakidos en peces depredadores. Tanto Rhamdia sp. como Parachromis sp., cumplen con estas características por su alta voracidad que puede influir también con la intensidad de infección y fungen como hospederos paraténicos, donde la L3 permanece en hipobiosis hasta alcanzar órganos y tejidos del hospedero definitivo que son aves $(3,4)$.

La intensidad de infección fue variable entre las zonas muestreadas. En Izabal, donde los peces mostraron mayor peso y longitud en comparación con los peces de Guanacaste, se observó una mayor carga parasitaria. Esto concuerda con el reporte de Mancinni et al (2) y Álvarez et al (17), que correlacionaron una alta intensidad de infección con una 
mayor edad, peso y talla en diferentes especies de peces, indicando que los especímenes más grandes poseen hábitos alimenticios más voraces y su edad prolongada les ha permitido estar expuestos con mayor frecuencia a más especímenes parasitarios por mayor tiempo, por consiguiente, poseen una mayor predisposición a ser parasitados por nemátodos del género Contracaecum.

También se observó, una alta tendencia de C. multipapillatum por ubicarse formando quistes en órganos viscerales, mientras que la cantidad de quistes adheridos en musculatura fue muy escasa. Estos resultados son similares a los hallados por Álvarez et al (17), Iglesias et al (18) y Hernández et al (19) que relacionan estas características con una baja capacidad de migración del parasito desde vísceras hacia musculatura una vez que el pez ha muerto. Esto es importante, pues determina la capacidad infectiva de las larvas para el ser humano al momento de consumir productos pesqueros.

Con respecto a los parámetros fisicoquímicos, cabe destacar que en los sitios con niveles de oxígeno inferiores a 2 ppm se observó una baja proporción de nemátodos en peces, lo que podría explicarse tomando en cuenta que los valores óptimos para la salud de un ecosistema varían entre 5 a 14 ppm $(20,21,22)$. La presencia de una baja presión de oxígeno puede originar condiciones de estrés que obliga a los organismos presentes en estos hábitats, a buscar zonas más favorables para su supervivencia. El bajo nivel de oxígeno puede estar condicionando la capacidad de desarrollo y éxito en la supervivencia de los helmintos con ciclos de vida acuáticos, por la presencia o ausencia de otros organismos acuáticos que actúen como sus hospederos, (22).

El presente estudio reporta por primera vez la presencia de Contracaecum multipapillatum c.l., un nemátodo zoonótico en peces de agua dulce de las regiones de la provincia de Guanacaste, Costa Rica y del departamento de Izabal, Guatemala, donde son comercializados artesanalmente y constituyen una fuente básica de alimentación para poblaciones cercanas a ríos y lagos. La presencia del nemátodo zoonótico en Centroamérica debe de alertar a los profesionales de la Salud para tomar en cuenta la Contracecosis como diagnóstico diferencial en casos clínicos donde los antecedentes incluyan hábitos de consumo de carne de pescado cruda o semicocinada. Se recomienda implementar políticas de prevención enfocadas a crear buenos hábitos de limpieza y cocina de platillos a base de carne de pescado y así prevenir infecciones accidentales.

\section{Conflictos de interés}

Los autores declaran no tener ningún conflicto de intereses.

\section{Agradecimientos}

Al Servicio Alemán de Intercambio Académico (DAAD) por la beca otorgada a Luis Choc para obtener la Maestría en Enfermedades Tropicales de la Universidad Nacional de Costa Rica (UNA), y por el financiamiento proporcionado para llevar a cabo la presente investigación. A Rolando Wer, al personal técnico de la Universidad Técnica Nacional (UTN) de Costa Rica, al Consejo Nacional de Áreas Protegidas (Guatemala), y al Centro de Estudios de Conservación (Guatemala) por su apoyo con el trabajo de campo y muestreo.

\section{REFERENCIAS}

1. Motamedi M, Iranmanesh A, Teimori A, Mahmoud S, Nasibi S. Detection of Contracaecum multipapillatum (Nematoda: Anisakidae) in the indigeneous killfish Aphanius hormuzensis (Teleostei; Aphaniidae) and its histopathological effects: A review or Iranian Aphanius species parasites. J Appl Ichthyol. 2018; 35(2):1-12. https://doi.org/10.1111/ jai.13848

2. Mancinni M, Biolé F, Salinas V, Guagliardo S, Tanzola R, Morra G. Prevalence, Intensity and Ecological aspects of Contracaecum sp. (Nematode: Anisakidae) in freshwater fish of Argentina. Neotrop. Helminthol. 2014; 8(1):111122. http://sisbib.unmsm.edu.pe/BVRevistas/neohel/v8n1/pdf/a10v8n1.pdf

3. García ML, Salguero G, García L, Osorio D, y Perez-Ponce G. Endohelminths of some species of fishes from Lake Xochimilco, México. Rev Mex Biodivers. 2016. 87(4):1360-1364. https://doi.org/10.1016/j.rmb.2016.06.018

4. Valle I, Molina D, Benítez R, Hernández S, Adroher F. Early developmentand life cycle of Contracaecum multipapillatum s.l. from a brown pelican Pelecanus occidentalis in the Gulf of California, Mexico. Dis Aquat Org. 2017; 125:167-178. https://doi.org/10.3354/dao03147 
5. Chiclla A, y Tantas D. Infection of Contracaecum sp. larvae (NEMATODA: ANISAKIDAE) in Tilapia (Oreochromis niloticus) of Perú. Biologys. 2015. 13(2):419-427. http://sisbib.unmsm.edu.pe/BVRevistas/biologist/v13 n2/pdf/ a21v13n2.pdf

6. Maniscalchi M, Lemus D, Marcano Y, Edgar N, Marianella Z, Narváez N. Larvas anisakidae en peces del género mugil comercializados en mercados de la región costera nor-oriental e insular de Venezuela. Saber. 2015; 27(1):30-38. http://www.scielo.org.ve/pdf/saber/v27n1/art05.pdf

7. Serrano E, Tantalean M, Quispe M, Casas G. Presencia de larvas de Contracaecum sp. (Nematoda, Anisakidae) en el pez Astronotus ocellatus, destinado al consumo humano en Loreto, Peru. Salud Tecnol Vet. 2015; 3(2):31-34. https://doi.org/10.20453/stv.v3i1.2757

8. Menghi C, Comunaleb E, Gattaa, C. Anisakiosis: primer diagnóstico en Buenos Aires, Argentina. Rev Soc Ven Microbiol. 2011; 31(1):71-73. http://190.169.30.98/ojs/index.php/rev vm/article/download/3087/2952

9. Menghi C, Gatta C, Arias L, Santonia G, Nicola F, Smayevsky J, et al. Human infection with Pseudoterranova cattani by ingestion of "ceviche" in Buenos Aires, Argentina. Rev Argent Microbiol. 2019; 1-3. https://doi.org/10.1016/j. $\underline{\text { ram.2019.06.005 }}$

10. Lim H, Jung B, Cho J, Yooyen T, Shin E, Chai J. Molecular Diagnosis of Cause of Anisakiasis in Humans, South Korea. Emerg. Infect. Dis. 2015; 21(2):342-344. https://doi.org/10.3201/eid2102.140798

11. Solas M, García M, de las Heras C. Anisakis simplex antigens in fresh and frozen-thawed muscle of anchovies in vinegar. Food Sci Technol Int. 2009. 15(2):139-148. https://doi.org/10.1177/1082013208105171

12. da Silva R, Penha A, Nascimento J, Guerreiro E. Contracaecum larvae: morphological and morphometric retrospective analysis, biogeographay and zoonotic risk in the amazona. Braz J Vet Parasitol. 2019; 28(1):12-32. http://doi. org/10.1590/S1984-29612019002

13. D'Amelio S, Cavallero S, Dronen N, Barros N, Paggi L. Two new species of Contracaecum Railliet and Henry 1912 (Nematoda: Anisakidae), C. fagerholmi n. sp. and C. rudolphii F from the brown pelican Pelecanus occidentalis in the northern Gulf of Mexico. Syst Parasitol. Syst Parasitol. 2012; 81(1):1-16. https://doi.org/10.1007/s11230-011$\underline{9323-\underline{x}}$

14. BautistaC,MonksS,PulidoG,RodríguezA.Revisiónbibliográficadealgunostérminosecológicosusadosenparasitología, y su aplicación en estudios de caso. En: Pulido G, Monks S, Lopez M, editores. Estudios en Biodiversidad. Vol 1. Lincoln: Zea E-Books; 2015. https://digitalcommons.unl.edu/cgi/viewcontent.cgi?article=1034\&context=zeabook

15. D’amico P, Malandra R, Costanzo F, Castigliego L, Guidi A, Gianfaldoni D, et al. Evolution of the Anisakis risk management in the European and Italian context. Food Res Int. 2014. 64:348-362. http://dx.doi.org/10.1016/j. foodres.2014.06.038

16. ÁlvarezA, Méndez Y, Reyes J, Romo C, Hernández G. Nematode parasites in the striped mullet (Mugil cephalus Linnaeus, 1758) in the southern Gulf of California. Lat Am J Aquat Res. 2020. 48(1):106-113. https://doi.org/10.3856/vol48issue1-fulltext-2326

17. Iglesias L, Benítez R, Adroher F, Valero A. Helminth infection in Mugil incilis from Cartagena de Indias, Colombian Caribbean coast. Helminthologia. 2011. 48(1):36-40. https://doi.org/10.2478/s11687-011-0007-5

18. Hernández J, Aznar F, Blasco I, García N, Vilora M, Crespo E, et al. Description, microhabitat selection and infection patterns of sealworm larvae (Pseudoterranova decipiens species complex, nematoda: ascaridoidea) in fishes from Patagonia, Argentina. Parasit Vectors. 2013. 6(252):1-15. https://doi.org/10.1186/1756-3305-6-252

19. Boyd C. Solar Radiation and Water Temperature. En Boyd, C. E. Water Quality: An Introduction (Segunda ed.). Londres, Inglaterra: Springer International Publishing. 2015. https://doi.org/10.1007/978-3-030-23335-8 2

20. Boyd C. Dissolved Oxygen and Other Gases. En Boyd, C. E. Water Quality: An Introduction (Segunda ed.). Londres, Inglaterra: Springer International Publishing. 2015. https://doi.org/10.1007/978-3-030-23335-8 7 
21. Boyd C. Carbon Dioxide, pH, and Alkalinity. En Boyd, C. E. Water Quality: An Introduction (Segunda ed.). Londres, Inglaterra: Springer International Publishing. 2015. https://doi.org/10.1007/978-3-030-23335-8 9

22. Monks S, Pulido G, Bautista C, Alemán B, Falcón J, Gaytán J. El uso de helmintos parásitos como bioindicadores en la evaluación de la calidad del agua: Lago de Tecocomulco vs. Laguna de Metztitlán, Hidalgo, México. En: Pulido G, Monks S, editores. Estudios científicos en el estado de Hidalgo y zonas aledañas. Vol 2. Lincoln: Zea E-Books; 2013. https://digitalcommons.unl.edu/cgi/viewcontent.cgi?article=1015\&context=zeabook 